\title{
Auto-ecologia de Hypsiboas albopunctatus (Anura, Hylidae) em área de Cerrado no sudeste do Brasil
}

\author{
Keni P. R. Muniz, Ariovaldo A. Giaretta, Wagner R. Silva \& Kátia G. Facure
}

Laboratório de Ecologia, Comportamento e Sistemática de Anuros Neotropicais, Universidade Federal de Uberlândia, 38400-902 Uberlândia, MG, Brasil. (thoropa@inbio.ufu.br)

\begin{abstract}
Autoecology of Hypsiboas albopunctatus (Anura, Hylidae) in an area of Cerrado in southeast of Brazil. Herein we present new data on the breeding activity of the Neotropical treefrog H. albopunctatus. Data was collected in the Municipality of Uberlândia, Minas Gerais State, Brazil. Behavioral events were observed focally. We determined the pattern of spatial distribution of the tadpoles. We compared abundance of tadpoles in lakes and swamps and correlated it with variation in depth, amount of vegetable substratum and number of arthropods through a Principal Component Analysis. We tested the palatability of eggs and tadpoles for two species of teleosts. Males vocalized in almost all months of the year. Males were smaller than the females and presented one spine in each prepolex. Amplexus was alternated between axillary and tympanic types. Clutches averaged 769 eggs and were laid floating in a single layer. Tadpole coloration changed ontogenetically from black to tan. Eggs were not consumed by the two tested species of teleosts, but the tadpoles yes. Tadpoles had an aggregated distribution in their habitats. The swamps presented larger number and mass of tadpoles. Only in the lakes there was a positive correlation between the abundance of tadpoles and depth, amount of plant substratum and arthropods. The almost continuous reproduction of $H$. albopunctatus along the year is a rare behavior among frogs of southeastern Brazil. Tadpoles were more abundant in the swampy probably due to absence of predators such as teleosts. Unpalatable eggs and the use of refuges by the tadpoles may be the mechanisms allowing reproduction of $H$. albopunctatus in habitats with predatory teleosts.
\end{abstract}

KEYWORDS. Hypsiboas albopunctatus, reproduction, abundance, teleosts, palatability.

RESUMO. Neste estudo, descrevemos aspectos da biologia reprodutiva de $H$. albopunctatus. Coletamos os dados no município de Uberlândia (MG), Brasil. Eventos comportamentais foram observados focalmente. Determinamos o padrão de distribuição espacial dos girinos. Comparamos a abundância de girinos em lagos e brejos, correlacionando-a com profundidade, quantidade de substrato vegetal e número de artrópodes através de uma Análise de Componentes Principais. Testamos a palatabilidade de ovos e girinos para duas espécies de teleósteos. A atividade de vocalização ocorria em praticamente todos os meses do ano. Os machos eram menores que as fêmeas e tinham um espinho em cada prepolex. O amplexo foi alternado entre os tipos axilar e timpânico. As desovas (média de 769 ovos) foram postas à superfície da água em uma monocamada. Girinos recentes eram pretos, porém tornavam-se acobreados ao longo do desenvolvimento. Diferentemente dos girinos, os ovos foram impalatáveis para os teleósteos testados. Os girinos eram mais abundantes nos brejos e se distribuíam de forma agregada no ambiente. Somente nos lagos houve correlação positiva entre a abundância de girinos e profundidade, número de artrópodes e quantidade de substrato vegetal. A reprodução da espécie quase continua ao longo do ano é um comportamento raro entre anuros do sudeste do Brasil. A abundância maior de girinos nos brejos pode ser devido à ausência de teleósteos predadores. A impalatabilidade dos ovos e o uso de micro-hábitat de refúgio pelos girinos provavelmente são os mecanismos que possibilitam a coocorrência de H. albopunctatus com teleósteos.

PALAVRAS-CHAVE. Hypsiboas albopunctatus, reprodução, abundância, teleósteos, palatabilidade.

Os hilídeos (Hylidae) exibem uma grande variedade de modos reprodutivos principalmente quanto ao microhábitat de oviposição. A maioria das espécies desse grupo deposita ovos diretamente na água, porém, algumas desovam em folhas suspensas sobre a água ou em câmaras subterrâneas (LuTZ, 1973; WeLls, 1977; CARDOSO \& HADDAD, 1984; HöDL, 1990; DuEllman \& TRUEB, 1994; Haddad \& Sawaya, 2000; Haddad \& Prado, 2005). Mesmo entre as espécies que desovam na água existem diferenças quanto ao micro-ambiente no qual os ovos são postos; algumas aderem ovos à vegetação subaquática enquanto que outras depositam ovos flutuantes em bacias escavadas no lodo (LuTz, 1960; Martins \& Haddad, 1988; Martins, 1993; Duellman \& Trueb, 1994; Haddad \& Prado, 2005).

Hypsiboas albopunctatus ocorre nas regiões central, sul e sudeste do Brasil, nordeste da Argentina, leste da Bolívia, Paraguai e norte do Uruguai (Frost, 2007). No presente estudo, descrevemos alguns aspectos da auto-ecologia de $H$. albopunctatus, tais como: ambiente e época de reprodução, comportamentos de corte e oviposição, tipos de amplexo, estrutura e número de ovos da desova e padrão de distribuição espacial dos girinos. Também testamos se fatores como tipo de corpo d'água, profundidade da água e substrato vegetal têm correlação com a abundância de girinos e se os ovos e girinos são palatáveis para duas espécies de teleósteos (Teleostei). Discutimos também a evolução de alguns caracteres comportamentais e ecológicos (e.g. tipo de desova) entre espécies de hilídeos.

\section{MATERIAL E MÉTODOS}

Realizamos o presente estudo no município de Uberlândia (1859’S; 48¹8’O; Minas Gerais, Brasil), localizado no domínio do Bioma Cerrado. A estação quente e úmida se estende de outubro a abril e a fria e seca abrange os meses de maio a setembro, sendo que no inverno podem ocorrer geadas (Rosa et al., 1991). Conduzimos as observações em lagos e brejos na reserva biológica do Clube Caça e Pesca e na periferia da cidade. 
Comparamos adultos de ambos os sexos quanto a diferenças do comprimento rostro-cloacal (CRC) e caracteres sexuais secundários. A significância das diferenças entre as médias foi testada através do teste-t (ZAR, 1999). As medições foram feitas com paquímetro $(0,1 \mathrm{~mm})$. Ovários e ovidutos hipertrofiados foram considerados como indicadores de maturidade das fêmeas; os machos medidos foram coletados vocalizando. O número de ovos por postura foi determinado com base em desovas e pela contagem de ovócitos ovarianos maduros ( $>1,4 \mathrm{~mm})$. Testamos se havia correlação entre o tamanho das fêmeas e o número de ovócitos pelo coeficiente de correlação de Pearson (ZAR, 1999).

Determinamos a duração da temporada de atividade vocal dos machos com base em dados que datam de agosto de 1998 a dezembro de 2005. As visitas ao campo foram semanais entre janeiro de 2002 e março de 2005. Realizamos as observações a partir do início do turno de vocalização ( $c a$. 18h e 30min) e acompanhamos casais em corte ou em amplexo focalmente, com registro de todos os comportamentos (MARTIN \& BATESON, 1986) até o macho liberar a fêmea. Consideramos como machos satélites aqueles indivíduos que permaneciam em silêncio e a menos de $15 \mathrm{~cm}$ de distância de machos que vocalizavam (WELLS, 1977).

Descrevemos a variação ontogenética na coloração dos girinos. Procuramos com peneira (malha $4 \mathrm{~mm}$ ) por girinos no inverno (final de junho) em locais nos quais foram observados machos cantando e desovas em meses anteriores. As referências aos estágios larvais seguiram GOSNER (1960).

Buscamos por eventuais diferenças na abundância e padrão de distribuição espacial de girinos da espécie em lagos e brejos. Uma diferença importante entre esses dois ambientes é que somente nos lagos existiam teleósteos potencialmente predadores de ovos e girinos (e.g. acarás Cichlasoma sp.). Realizamos as 70 amostragens dentro de parcelas, sendo que 45 foram em três lagos e 25 em três brejos. Nos lagos, as parcelas foram estabelecidas nas bordas a até três metros da margem. Essas parcelas consistiam em um anel de metal com alças (56cm diâmetro, $45 \mathrm{~cm}$ altura), o qual era introduzido rapidamente no local a ser amostrado. Após a introdução checávamos se a vedação do fundo impedia a fuga de girinos. De cada parcela medimos a profundidade da água e removemos com peneira (malha $4 \mathrm{~mm}$ ) o substrato vegetal, artrópodes e girinos, os quais foram acondicionados em sacos plásticos rotulados e preservados em formalina (5\%). A massa dos girinos (balança $0,01 \mathrm{~g}$ ) por parcela foi determinada depois de enxugar cada indivíduo com papel toalha. Para descrever a variação na profundidade da água, massa de substrato vegetal (desidratado a $90^{\circ} \mathrm{C}$ até peso constante) e número de artrópodes entre as parcelas foi feita uma análise de componentes principais (ACP) sobre uma matriz de correlação desses parâmetros (MANLY, 1986). Para avaliar o efeito dessas variáveis combinadamente sobre a abundância de girinos, testamos a correlação entre o número de girinos em cada parcela e os escores dessas no primeiro eixo da ACP, usando o coeficiente de correlação de Spearman (ZAR, 1999). Diferenças nas medianas da abundância e massa de girinos, profundidade, quantidade de substrato vegetal e número de artrópodes entre os dois tipos de corpos d'água foram testadas pelo teste U de Mann-Whitney (ZAR, 1999).

Determinamos o padrão de distribuição espacial dos girinos com base no Índice de Dispersão (ID; relação variância/média), derivado dos dados de densidade obtidos nas parcelas (anel de metal) e no cálculo de $\chi^{2}$ (programa Negbinom: KREBS, 1989). Checamos se o padrão agregado observado para os dois tipos de corpos d'água (brejos e lagos) adequou-se ao modelo teórico de distribuição binomial negativa (BN) através do teste T de adequação de ajuste (programa Anscombe: KREBS, 1989). A hipótese nula (BN descreve adequadamente as distribuições) foi aceita quando o valor observado de $\mathrm{T}$ foi menor que duas vezes o do seu erro-padrão (EP). Os intervalos de confiança para a densidade média (95\%) foram determinados de acordo com esse modelo. Nos brejos a densidade média dos girinos foi estimada de acordo com o modelo $\mathrm{BN}$, porque o valor observado ultrapassou o limite superior do intervalo de confiança da média (programa Negbinom: KREBS, 1989).

Testamos a palatabilidade de ovos e girinos para duas espécies de teleósteos ciclídeos: seis indivíduos de acarás (Cichlasoma sp.) e seis de óscars, Astronotus ocellatus (Agassiz, 1831). Os acarás (ca.50mm CP) foram coletados nos lagos onde $H$. albopunctatus se reproduz e os óscars (ca.60mm CP) foram adquiridos no comércio. Conduzimos os testes em recipientes plásticos marrons (81) com três litros de água declorificada, mantidos em local sombreado sob condições ambientais de fotoperíodo e temperatura $\left(29^{\circ} \mathrm{C}\right)$. Conforme Hero et al . (2001), assumimos que, depois de 24 horas, o consumo de ovos ou girinos abaixo de $30 \%$ indicaria impalatabilidade. Como controle, utilizamos outras duas espécies de anuros: ovos de Physalaemus cuvieri Fitzinger, 1826 (Leiuperidae) ( $0,9 \mathrm{~mm}$; cor creme) e girinos de Odontophrynus cultripes Reinhardt \& Lütken, 1862 (Cycloramphidae) (estágio 25; < 30mm CT). Antes dos testes, os teleósteos foram submetidos a um jejum de 48 horas. Em cada tratamento $(\mathrm{n}=3$ replicações para cada espécie de teleósteo) ou controle colocamos um teleósteo e 20 ovos ( $n=3$ desovas $)$ ou cinco girinos de diferentes desovas. Testamos a palatabilidade dos girinos de $H$. albopunctatus na fase em que são pretos (estágio 23; $<15 \mathrm{~mm}$ CT) e quando apresentam coloração acobreada (estágio 25).

Acarás (AAG-UFU 3212, 3213), adultos e girinos de H. albopunctatus (AAG-UFU: 2195; 2108; 2213; 2104; 1991) estão depositados na Coleção Zoológica AAG-UFU do Museu de Biodiversidade do Cerrado (Universidade Federal de Uberlândia, Uberlândia, Minas Gerais, Brasil).

\section{RESULTADOS}

Os machos adultos (média $\mathrm{CRC}=44,8 \mathrm{~mm}$; DP $=$ 3,1; Amplitude $=40,0-50,3 ; \mathrm{n}=18)$ de $H$. albopunctatus foram menores (teste-t $=2,57 ; \mathrm{p}=0,01$ ) que as fêmeas (média $=48,1 \mathrm{~mm} ; \mathrm{DP}=4,5 ;$ Amplitude $=40,9-55,6 ; \mathrm{n}=$ 18). Os machos possuíam um espinho em cada prepólex.

Os machos vocalizaram em praticamente todos os meses, com exceção de julho. Encontramos quatro fêmeas com ovócitos ovarianos maduros entre os meses de 
novembro e janeiro e 10 desovas entre novembro e maio. $\mathrm{Na}$ estação seca (junho), encontramos girinos recentes (estágio 23; <20mm CT) e tardios (estágio 25-30; 35-50mm $\mathrm{CT})$. Juvenis $(\mathrm{CRC}<25 \mathrm{~mm} ; \mathrm{n}=15)$ foram encontrados entre os meses de abril e dezembro.

A atividade de vocalização e desovas ocorreram às margens de corpos d'água permanentes com água corrente, mesmo que fraca. Machos vocalizavam no chão $(n=11)$ ou na vegetação a até dois metros de altura $(n=$ 5). As vocalizações iniciavam-se pouco antes do ocaso (ca. 18h), podendo se estender até às $3 \mathrm{~h}$. De 15 machos em atividade de canto examinados, seis estavam acompanhados por machos satélites, mas não observamos combates físicos entre eles.

Observamos cinco vezes comportamentos de corte e desova. A fêmea aproximava-se de um macho em atividade de vocalização e este, ao perceber sua presença $(<10 \mathrm{~cm})$, se dirigia até ela. Quando juntos, a fêmea tocava o macho com o focinho e, em seguida, era amplexada nos ângulos da maxila (amplexo timpânico) (Fig. 1). O casal podia permanecer imóvel por até 40 minutos, após o que a fêmea se movia em direção à água. No local de desova, a fêmea mergulhava (por $6 \mathrm{~s}$ ) periodicamente e, em cada mergulho, girava em torno de si mesma, afastando a vegetação com as pernas; durante os mergulhos o macho trocava o amplexo de timpânico para axilar. Os mergulhos e o afastamento da vegetação podiam durar toda a madrugada. No momento da liberação dos ovos, a fêmea elevava a cloaca até pouco acima da linha d'água; a liberação de todos os ovos durava cerca de 16 minutos. Depois de finalizada a postura, o macho liberava a fêmea e os dois abandonavam o local. Em um dos casos, um macho que cantava na vegetação foi até uma fêmea que estava no chão. Depois do toque dela, ele foi em direção à água emitindo cantos de anúncio e foi seguido por ela $(1,5 \mathrm{~m})$; eles entraram em amplexo à beira da água, próximo $(30 \mathrm{~cm})$ ao local onde ocorreu a desova.

As desovas consistiam em uma monocamada de ovos flutuantes quase circular, com diâmetro aproximado de $15 \mathrm{~cm}(\mathrm{n}=10)$ (Fig. 2). Elas foram encontradas em locais rasos (5-40cm profundidade), geralmente em meio à vegetação emergente $(n=10)$. O local de postura geralmente apresentava continuidade hídrica com o corpo d'água maior $(\mathrm{n}=8)$, porém, em dois casos, as desovas

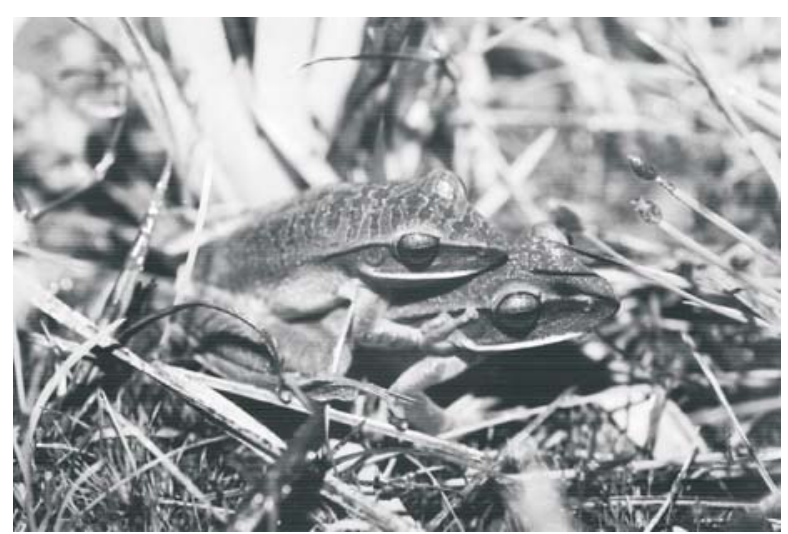

Fig. 1. Casal de Hypsiboas albopunctatus em amplexo, momentos antes da fêmea se dirigir para a água. Note que a posição do amplexo é timpânica, com discos adesivos do macho tocando o tímpano da fêmea. Espécimes de Uberlândia, MG, Brasil. Macho (42,4mm CRC), fêmea $(50,0 \mathrm{~mm})$. foram postas em depressões no solo encharcado (Fig. 2) e a 30-100cm de distância do corpo d'água principal. Essas depressões tinham tamanho coincidente com o diâmetro das desovas. As desovas possuíam em média 769 ovos $(\mathrm{DP}=120 ;$ amplitude $=552-979 ; \mathrm{n}=6)$ e geralmente recebiam luz solar direta $(n=7)$. Cada ovo possuía um pólo escuro e um outro amarelo pálido; a parte vitelínica tinha em média $1,6 \mathrm{~mm}(\mathrm{DP}=0.0)$ de diâmetro e, considerando-se a cápsula gelatinosa, $2,2 \mathrm{~mm}(\mathrm{n}=30$ ovos, 6 desovas). As fêmeas analisadas tinham em média 744 ovócitos ovarianos maduros ( $\mathrm{DP}=177 ; \mathrm{n}=4)$; houve correlação marginalmente significativa entre o tamanho das fêmeas e o número de ovócitos ( $\mathrm{r}=0,92 ; \mathrm{p}=0,084$; $\mathrm{n}=4$ ).

Girinos pequenos ( $<15 \mathrm{~mm}$ CT; estágio 23) eram pretos, porém depois de dez dias tornavam-se acobreados (estágio 25). Girinos na iminência da metamorfose (estágio $42 ; n=2)$ e os recém-metamorfoseados $(n=5)$ eram esverdeados (estágio 46). O maior girino (estágio 42) coletado tinha $62,7 \mathrm{~mm}$ de comprimento total e o menor recém-metamorfoseado, $16 \mathrm{~mm}$ de CRC.

Os girinos (14-50mm CT) se distribuíram de forma agregada nos brejos (ID $=34,5 ; \chi^{2}=828 ; \mathrm{gl}=24 ; \mathrm{p}<$ 0,$001 ; \mathrm{n}=25$ parcelas) e lagos (ID $=12,1 ; \chi^{2}=534 ; \mathrm{gl}=44$; $\mathrm{p}<0,001 ; \mathrm{n}=45)$. A distribuição Binomial Negativa descreveu adequadamente o padrão agregado dos dois tipos de corpos d'água (Brejos: T = -975; EP = 5262; Lagos: $\mathrm{T}=-37 ; \mathrm{EP}=156)$. Os pontos amostrados nos dois tipos de corpos d'água não diferiram entre si nos parâmetros quantidade de substrato vegetal e profundidade da água (Tab. I). Os brejos apresentaram uma densidade média de girinos entre 2,9 (estimada, Binomial Negativa) e 6,6 vezes maior (5,4 mais em biomassa) que os lagos e maior quantidade de artrópodes (Tab. I). Na amostra global, a densidade de girinos se correlacionou com a de artrópodes $\left(r_{s}=0,57\right.$; $\mathrm{p}<0,001 ; \mathrm{n}=70$ parcelas), mas não com a quantidade de substrato vegetal $\left(r_{\mathrm{s}}=0,19 ; \mathrm{p}>0,05 ; \mathrm{n}=70\right)$ nem com profundidade $\left(r_{s}=0,20 ; p>0,05 ; n=70\right)$.

Analisando os dados separadamente por ambiente (lagos ou brejos), somente nos lagos houve correlação entre o número de girinos e profundidade da água $\left(r_{\mathrm{s}}=0,27 ; \mathrm{p}<0,05 ; \mathrm{n}=45\right)$, quantidade de substrato vegetal $\left(r_{s}=0,25 ; p<0,05 ; n=45\right)$ e artrópodes $\left(r_{s}=0,44 ; p<0,005\right.$;

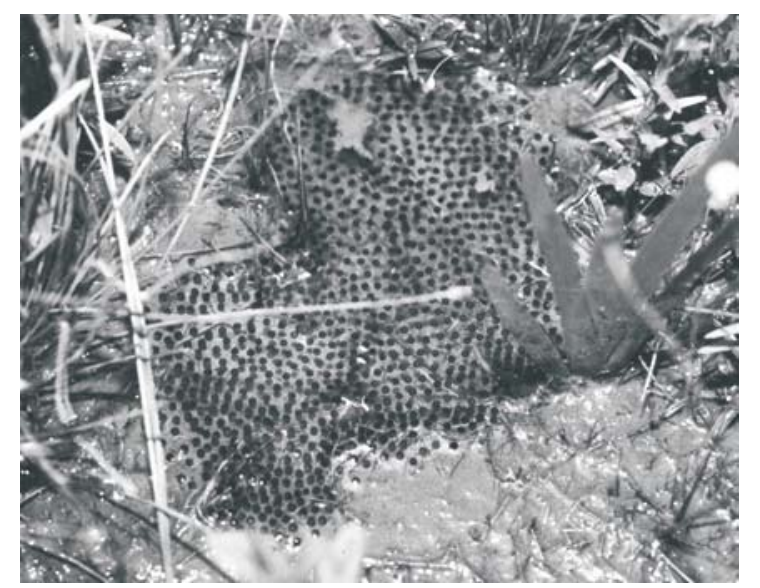

Fig. 2. Desova de Hypsiboas albopunctatus. Note que os ovos estão dispostos em uma monocamada flutuante. Esta desova estava isolada $(30 \mathrm{~cm})$ do corpo d'água principal. Uberlândia, MG, Brasil. 
$\mathrm{n}=45)$. O primeiro eixo da Análise de Componentes Principais explicou 49,6\% da variância dos dados e a variável mais importante nesse eixo foi a quantidade de substrato vegetal (autovetor $=0,687$ ) (Fig. 3). O segundo eixo explicou $31,6 \%$ e as variáveis mais importantes foram artrópodes (autovetor $=-0,759$ ) e profundidade (autovetor $=0,651)$. O número de girinos se correlacionou com os escores das parcelas no primeiro eixo $\left(\mathrm{r}_{\mathrm{s}}=0,42 ; \mathrm{p}\right.$ $<0,001 ; \mathrm{n}=70)$. Ao se considerar cada tipo de corpo d'água em separado, o número de girinos nos lagos se correlacionou com os escores das parcelas no primeiro eixo $\left(\mathrm{r}_{\mathrm{s}}=0,42 ; \mathrm{p}<0,005 ; \mathrm{n}=45\right)$, porém nos brejos não houve essa correlação $\left(r_{s}=0,11 ; p>0,50 ; n=25\right)$.

Tabela I. Abundância de girinos e parâmetros das variáveis ambientais de dois tipos de corpos d'água utilizados para reprodução por Hypsiboas albopunctatus em Uberlândia, Minas Gerais, Brasil. Lagos ( $n=45$ parcelas) representaram locais com presença de teleósteos potencialmente predadores; Brejos $(n=25)$ eram corpos d'água sem estes predadores. IC, Intervalo de confiança da média, segundo ajuste à distribuição de freqüências Binomial Negativa.

\begin{tabular}{lcccrr}
\hline & $\begin{array}{c}\text { Número } \\
\text { girinos }\end{array}$ & $\begin{array}{c}\text { Massa } \\
\text { girinos }(\mathrm{g})\end{array}$ & $\begin{array}{c}\text { Profundidade } \\
(\mathrm{cm})\end{array}$ & $\begin{array}{r}\text { Vegetal } \\
(\mathrm{g})\end{array}$ & $\begin{array}{r}\text { Número } \\
\text { Artrópodes }\end{array}$ \\
\hline Lagos & & & & & \\
Mínimo & 0,0 & 0,0 & 5,5 & 0,0 & 0,0 \\
Máximo & 29,0 & 15,0 & 36,0 & 86,2 & 15,0 \\
Mediana & 0,0 & 0,0 & 12,0 & 21,4 & 0,0 \\
Média & 1,8 & 0,8 & 14,5 & 26,8 & 1,5 \\
Variância & 21,6 & 2,6 & 7,4 & 26,1 & 2,7 \\
95\% IC & $0,4-3,2$ & & & & \\
Média (BN) & & & & & \\
$1^{\circ}$ quartil & 0,0 & 0,0 & 9,4 & 3,6 & 0,0 \\
$3^{\text {o quartil }}$ & 2,0 & 0,5 & 18,2 & 42,3 & 2,0 \\
Brejos & & & & & \\
Mínimo & 0,0 & 0,0 & 0,0 & 0,0 & 0,0 \\
Máximo & 99,0 & 25,9 & 33,0 & 149,7 & 15,0 \\
Mediana & 4,5 & 2,0 & 15,5 & 21,9 & 5,0 \\
Média & 11,8 & 4,3 & 14,9 & 36,0 & 5,0 \\
Variância & 408,7 & 6,5 & 8,4 & 38,2 & 4,3 \\
Média & 5,3 & & & & \\
Estimada (BN) & & & & & \\
95\% IC & $2,5-10,8$ & & & & \\
Média (BN) & & & & & \\
$1^{\circ}$ quartil & 3,0 & 0,7 & 7,5 & 9,4 & 1,0 \\
$3^{\text {o quartil }}$ & 13,0 & 4,3 & 19,0 & 60,6 & 8,0 \\
Teste U & 961 & 914 & 609 & 649 & 894 \\
p & $<0,001$ & $<0,001$ & 0,57 & 0,29 & $<0,001$ \\
\hline
\end{tabular}

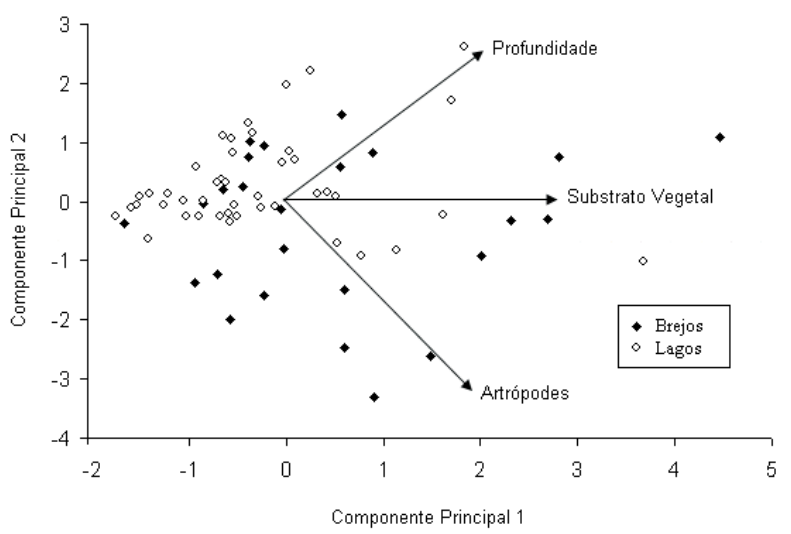

Fig. 3. Posição das parcelas $(n=70)$ em dois primeiros eixos da Análise de Componentes Principais sobre as variáveis profundidade, número de artrópodes e substrato vegetal (massa seca) de dois tipos de ambientes de reprodução de Hypsiboas albopunctatus. Dados de Uberlândia, MG, Brasil.
Os ovos de H. albopunctatus não foram consumidos (> 95\% de rejeição) pelas duas espécies de teleósteos testadas, mas os de P. cuvieri sim (> 85\% consumidos). A maioria (>94\%) dos girinos $H$. albopunctatus e $O$. cultripes (controle) foi ingerida por ambas as espécies de teleósteos. Todos os girinos de $H$. albopunctatus com coloração preta foram consumidos pelos acarás e $40 \%$ pelos óscars.

\section{DISCUSSÃO}

Na população estudada, os tamanhos de machos e fêmeas foram semelhantes aos apresentados por DE SÁ (1995), porém, foram menores que os de algumas populações descritas por LuTZ (1973); os fatores correlatos a essas diferenças regionais ainda precisam ser investigados. As fêmeas foram maiores que machos, o que é o padrão da maioria dos anuros (SHINe, 1979).

Como indicado pelo período de vocalização e presença de desovas e girinos pequenos $(<10 \mathrm{~mm})$, a atividade reprodutiva de $H$. albopunctatus no Cerrado é quase contínua ao longo do ano (BARRETo \& MoreIRA, 1996; presente estudo), comportamento raro entre os anuros do sudeste do Brasil (Pombal, 1997). Provavelmente, isto é condicionado pelo fato desta espécie se reproduzir em corpos d'água permanentes. Diferenças populacionais na duração da temporada reprodutiva da espécie podem estar relacionadas ao rigor e duração da estação fria e seca em diferentes localidades, com as populações em latitudes maiores apresentando temporadas reprodutivas mais curtas (CARDoso \& Haddad, 1992; Bernarde \& KoKubum, 1999).

Entre os anuros, a territorialidade geralmente é observada em espécies com reprodução prolongada e naquelas cujos machos mantêm sítios de vocalizações fixos (WeLLS, 1977). No entanto, não observamos combate físico entre machos de H. albopunctatus, como descrito para outras espécies do gênero e do grupo de $H$. albopunctatus (e.g. Guimarães et al., 2001; MENin et al., 2004). Como outros hilíneos (HADDAD, 1991; BASTOS \& Haddad, 1996; Guimarães \& Bastos, 2003), machos de $H$. albopunctatus adotam comportamento satélite e devem tentar interceptar fêmeas atraídas pelo macho em atividade vocal.

Em H. albopunctatus, o amplexo foi alternado entre os tipos timpânico e axilar durante a preparação dos locais de desova e a oviposição. A distinção que fizemos pode ser importante, pois parece indicar que a alternância está relacionada à presença do espinho no prepólex, o qual poderia machucar a fêmea durante as manobras para ajuste do local de oviposição. Martins (1993) também discute que a posição do amplexo em $H$. faber (WiedNeuwied, 1821) evita ferimentos na fêmea.

O grupo de $H$. albopunctatus foi definido em bases moleculares (FAIVOVICH et al., 2005), porém ainda não se conhecem derivações morfológicas, comportamentais ou ecológicas suportando seu monofiletismo. As espécies de Hypsiboas podem por ovos em uma massa aderida à vegetação subaquática (e.g. H. prasinus (Burmeister, 1856), H. goianus (Lutz, 1968)) ou em uma monocamada de ovos flutuantes (e.g. H. albopunctatus, H. fasciatus (Günther, 1858), H. lanciformis (Cope, 1871)) (LuTz, 1960, 
1973; CRUMP, 1974; HöDL, 1990; AICHINGER, 1992; MARTINS, 1993; RodríGueZ \& Duellman, 1994; Menin et al., 2004; HADDAD \& Prado, 2005; presente estudo). Se o gênero Hypsiboas representar um grupo monofilético, a condição "desova em monocamada flutuante" pode representar uma derivação que poderia indicar um subgrupo no gênero, o qual incluiria as espécies do grupo de $H$. albopunctatus e outras como $H$. faber e $H$. boans (Linnaeus, 1758), separadas daquelas com desova subaquática.

Diferentemente dos girinos, os ovos de $H$. albopunctatus foram impalatáveis para os teleósteos testados. Essa rejeição de ovos por parte de teleósteos também foi demonstrada para outras espécies de anuros da Amazônia (Magnusson \& Hero, 1991). Os girinos atingiram maiores densidades (e biomassa) nos brejos, nos quais os teleósteos potencialmente predadores estavam ausentes. A correlação positiva encontrada em lagos entre o número de girinos e as variáveis substrato vegetal e profundidade da água indica que essas características do hábitat podem estar relacionadas com a oferta de refúgio para os girinos contra predadores (e.g. teleósteos).

Fatores como competição, interação com predadores e disponibilidade de refúgio influenciam a sobrevivência de girinos e a impalatabilidade destes tem sido apontada como um dos principais mecanismos de defesa contra teleósteos (HEYER et al., 1975; MCDIARMID \& Altig, 1999; Hero et al., 2001). No entanto, em $H$. albopunctatus, a impalatabilidade não deve ser o fator condicionante da co-ocorrência de girinos e teleósteos. Como em outros anuros (MORIN, 1986; RESETARITS JR. \& Wilbur, 1989; Magnusson \& Hero, 1991; Semlitsch \& Reyer, 1992; Hero et al., 2001; BincKley \& ResetARITS JR., 2002; BinCKLEY \& RESETARITS JR., 2003; RiEGER et al., 2004), em H. albopunctatus a ação conjunta entre seleção de micro-sítios de oviposição e a impalatabilidade devem favorecer a sobrevivência das desovas e o uso de microhábitat de refúgio, a dos girinos.

Agradecimentos. Bolsa (AAG) e apoio financeiro do CNPq e FAPEMIG; bolsa CAPES (WRS e KGF). A. M. de S. Siqueira pelas críticas ao manuscrito. J. C. Oliveira-Filho auxiliou nos trabalhos de campo. M. Menin forneceu algumas referências bibliográficas. Os diretores do Clube Caça e Pesca autorizaram as atividades de campo na reserva do clube.

\section{REFERÊNCIAS BIBLIOGRÁFICAS}

AichINGER, M. 1992. Fecundity and breeding sites of an anuran community in a seasonal tropical environment. Studies on Neotropical Fauna and Environment 27(1):9-18.

Barreto, L. \& Moreira, G. 1996. Seasonal variation in age structure and spatial distribution of a savanna larval anuran assemblage in Central Brazil. Journal of Herpetology 30(1):87-92.

Bastos, R. P. \& Haddad, C. F. B. 1996. Breeding activity of the Neotropical treefrog Hyla elegans (Anura, Hylidae). Journal of Herpetology 30(3):355-360.

Bernarde, P. S. \& Kokubum, M. N. C. 1999. Anurofauna do município de Guararapes, Estado de São Paulo, Brasil (Amphibia: Anura). Acta Biológica Leopoldensia 21(1): 89-97.

Binckley, C. A. \& Resetarits JR., W. J. 2002. Reproductive decisions under threat of predation: squirrel treefrog (Hyla squirella) responses to banded sunfish (Enneacanthus obesus). Oecologia 130:157-161.
BinCKLey, C. A. \& Resetarits JR., W. J. 2003. Functional equivalence of non-lethal effects: generalized fish avoidance determines distribution of gray treefrog, Hyla chrysoscelis, larvae. Oikos 102:623-629.

Cardoso, A. J. \& Haddad, C. F. B. 1984. Variabilidade acústica em diferentes populações e interações agressivas de Hyla minuta (Amphibia, Anura). Ciência \& Cultura 36(8):1393-1399.

Cardoso, A. J. \& Haddad, C. F. B. 1992. Diversidade e turno de vocalizações de anuros em comunidade Neotropical. Acta Zoológica Lilloana 41:93-105.

Crump, M. L. 1974. Reproductive strategies in a tropical anuran community. University of Kansas Publications, Museum of Natural History. Miscellaneous publication No 61. $68 \mathrm{p}$.

De SÁ, R. O. 1995. Hyla albopunctata Spix, 1824. Catalogue of American Amphibians and Reptiles 602:1-5.

Duellman, W. E. \& Trueb, L. 1994. Biology of amphibians. Baltimore, The Johns Hopkins University Press. 670p.

Faivovich, J.; Haddad, C. F. B.; Garcia, P. C. A.; Frost, D. R.; Campbell, J. A. \& Wheeler, W. C. 2005. Systematic review of the frog family Hylidae, with special reference to Hylinae: phylogenetic analysis and taxonomic revision. Bulletin of the American Museum of Natural History New York (294): 1-240.

Frost, D. R. 2007. Amphibian species of the world 5.0, an online reference. The American Museum of Natural History. Disponível em: <http://research.amnh.org/herpetology/ amphibia/index.php>. Acesso em: 09.08.2007.

Gosner, K. L. 1960. A simplified table for staging anuran embryos and larvae with notes on identification. Herpetologica (16): $183-190$

Guimarães, L. D. \& Bastos, R. P. 2003. Vocalizações e interações acústicas em Hyla raniceps (Anura, Hylidae) durante a atividade reprodutiva. Iheringia, Série Zoologia, 93(2):149-158.

Guimarães, L. D.; Juliano, R. F. \& Bastos, R. P. 2001. Hyla raniceps. Combat. Herpetological Review 32(2):102-103.

Haddad, C. F. B. 1991. Satellite Behavior in the Neotropical Treefrog Hyla minuta. Journal of Herpetology 25(2):226-229.

Haddad, C. F. B. \& SawayA, R. J. 2000. Reproductive modes of Atlantic forest hylid frogs: a general overview and the description of a new mode. Biotropica 32(46):862-871.

Haddad, C. F. B. \& Prado, C. P. A. 2005. Reproductive modes in frogs and their unexpected diversity in the Atlantic Forest of Brazil. BioScience 55(3):207-217.

Hero, J. M.; Magnusson, W. E.; Rocha, C. F. D. \& Catterall, C. P. 2001. Antipredator defenses influence the distribution of amphibian prey species in the central Amazon rain forest. Biotropica 33(1):131-141.

Heyer, R. W.; McDiarmid, R. W. \& Weigman, D. L. 1975. Tadpoles, predation and pond habitats in the tropics. Biotropica 7(2): 100-111.

HöDL, W. 1990. Reproductive diversity in Amazonian lowland frogs. In: Hanke, W. ed. Biology and Physiology of the Amphibians. New York. v.38, p.41-60.

KreBs, C. J. 1989. Ecological methodology. New York, Harper and Row. 654p.

Lutz, B. 1960. The clay nests of Hyla pardalis Spix, 1824. Copeia 1960(4):364-366.

Lutz, B. 1973. Brazilian Species of Hyla. Austin, London, University of Texas Press. 260p.

Magnusson, W. E. \& Hero, J. M. 1991. Predation and the evolution of complex oviposition behaviour in Amazon rainforest frogs. Oecologia (86):310-318

Manly, B. F. J. 1986. Multivariate statistical methods. New York, A Primer Chapman and Hall. 159p.

Martins, M. 1993. Observations on the reproductive behavior in the Smith frog, Hyla faber. Herpetological Journal 3:31-34.

Martins, M. \& Haddad, C. F. B. 1988. Vocalizations and reproductive behavior in the smith frog, Hyla faber Wied (Amphibia: Hylidae). Amphibia-Reptilia 9:409-460.

Martin, P. \& BAteson, P. 1986. Measuring behaviour: an introductory guide. Cambridge, Cambridge University Press. $200 p$.

McDiarmid, R. W. \& Altig, R. 1999. Tadpoles: the biology of anuran larvae. The University of Chicago. 444p. 
Menin, M.; Silva, R. A \& Giaretta, A. A. 2004. Reproductive biology of Hyla goiana (Anura, Hylidae). Iheringia, Série Zoologia, 94(1):49-52.

Morin, P. J. 1986. Interactions between intraspecific competition and predation in an amphibian predator-prey system. Ecology 67:713-720

Pombal, JR. J. P. 1997. Distribuição espacial e temporal de anuros (Amphibia) em uma poça permanente na Serra de Paranapiacaba, sudeste do Brasil. Revista Brasileira de Biologia 57(4):583-594.

Resetarits JR., W. J. \& Wilbur, H. M. 1989. Choice of oviposition site by Hyla chrysoscelis: role of predators and competitors. Ecology 70(1):220-228.

Rieger, J. F.; Binckley, C. A. \& Resetarits JR., W. J. 2004. Larval performance and oviposition site preference along a predation gradient. Ecology 85(8):2094-2099.
Rodríguez, L. O. \& Duellman, W. E. 1994. Guide to the frogs of the Iquitos region, Amazonian Peru. The University of Kansas, Natural History Museum, Special Publication 22. $80 \mathrm{p}$.

Rosa, R.; Lima, S. C. \& Assunção, W. L. 1991. Abordagem preliminar das condições climáticas de Uberlândia (MG). Sociedade \& Natureza 3:91-108.

Semlitsch, R. D. \& Reyer, H. U. 1992. Modification of antipredator behavior in tadpoles by environmental conditioning. Journal of Animal Ecology 61:353-360.

Shine, R. S. 1979. Sexual selection and sexual dimorphism in the Amphibia. Copeia 1979(2):297-306.

WeLLS, K. D. 1977. The social behaviour of anuran amphibians. Animal Behavior 25:666-693.

ZAR, J. H. 1999. Bioestatistical analysis. 4 ed. New Jersey, Prentice- Hall. 929p 